INPLASY

PROTOCOL

To cite: Xiong et al. Evaluation of the Efficacy of Stem Cell Therapy in Ovariectomized Osteoporotic Rats Based on Micro-CT and Dual-energy Xray Absorptiometry: $A$ Systematic Review and MetaAnalysis. Inplasy protocol 202150017. doi:

10.37766/inplasy2021.5.0017

Received: 04 May 2021

Published: 04 May 2021

Corresponding author:

Zhencheng Xiong

xiongzc0527@163.com

Author Affiliation:

Institute of Medical

Technology, Peking University

Health Science Center, Beijing

100191, China

Support: Z181100001818006.

Review Stage at time of this submission: Data analysis.

Conflicts of interest:

None declared.

\section{Evaluation of the Efficacy of Stem Cell Therapy in Ovariectomized Osteoporotic Rats Based on Micro-CT and Dual-energy X-ray Absorptiometry: A Systematic Review and Meta-Analysis}

Xiong, Z1; Yi, P2; Zhang, C³.

Review question / Objective: The purpose of this work is to carry out a systematic review and meta-analysis of the efficacy of stem cell therapy in ovariectomized (OVX) osteoporotic rats.

Condition being studied: Osteoporosis is an abnormal bone metabolism disease characterized by microstructural degeneration of bone tissue and reduction in bone mass, resulting in increased brittleness of bone tissue and susceptibility to fracture. Due to the tissue regenerative potential of stem cell transplantation, it is now used in the treatment of various disease models such as osteoporosis. The purpose of this work is to carry out a systematic review and meta-analysis of the efficacy of stem cell therapy in ovariectomized (OVX) osteoporotic rats.

INPLASY registration number: This protocol was registered with the International Platform of Registered Systematic Review and Meta-Analysis Protocols (INPLASY) on 04 May 2021 and was last updated on 04 May 2021 (registration number INPLASY202150017).

\section{INTRODUCTION}

Review question / Objective: The purpose of this work is to carry out a systematic review and meta-analysis of the efficacy of stem cell therapy in ovariectomized (OVX) osteoporotic rats.
Condition being studied: Osteoporosis is an abnormal bone metabolism disease characterized by microstructural degeneration of bone tissue and reduction in bone mass, resulting in increased brittleness of bone tissue and susceptibility to fracture. Due to the tissue regenerative 
potential of stem cell transplantation, it is now used in the treatment of various disease models such as osteoporosis. The purpose of this work is to carry out a systematic review and meta-analysis of the efficacy of stem cell therapy in ovariectomized (OVX) osteoporotic rats.

\section{METHODS}

Participant or population: Ovariectomized (OVX) osteoporotic rats.

Intervention: This meta-analysis compares the effects of stem cells and OVX control and sham-operation group.

Comparator: The sources of stem cells are divided into four main categories, bone marrow mesenchymal stem cells (BMSCs), adipose-derived stem cells (ADSCs), amniotic membrane mesenchymal stem cells (AM-MSCs), and human umbilical cord blood-derived mesenchymal stem cells (hUCB-MSCs).

Study designs to be included: Randomized controlled trial (RCT) or non-RCT.

Eligibility criteria: All retrieved articles were screened by our research team according to the inclusion and exclusion criteria developed by the subject of this metaanalysis. Inclusion criteria included: (1) all studies involved comparing the effects of stem cell therapy to OVX control group or sham-operated group; (2) all included studies could be of either randomized controlled trial (RCT) or non-RCT; (3) the animal model was rat and osteoporosis model establishment was achieved by ovariectomy; (4) the source of stem cells was not limited; (5) the data of outcome measurements were obtained by micro-CT; (6) The data related to the outcome measurements could be successfully extracted.

Information sources: After identifying the topics for this meta-analysis, in order to obtain all relevant studies, two researchers from our research team each independently searched multiple databases according to the Cochrane Collaboration guidelines, including PubMed (1966 to April 1, 2021), Cochrane library (1966 to April 1, 2021), ScienceDirect (1980 to April 1, 2021), Embase (1980 to April 1, 2021), CNKI (1980 to April 1, 2021), and Wanfang Database (1980 to April 1, 2021). Literature search is achieved by concatenating MeSH terms and corresponding keywords using Boolean operators (AND or OR), including "stem cell", "mesenchymal stem cell or MSC", "bone marrow-derived mesenchymal stem cell or BMSC", "adipose-derived stem cell or ADSC", "osteoporosis", "ovariectomized or OVX", and "rat".

Main outcome(s): Bone mineral density (BMD) was the primary outcome measurement, and bone volume/total volume (BV/TV), trabecular number (Tb.N), trabecular thickness (Tb.Th) and trabecular spacing (Tb.Sp) were the secondary outcome measurement.

Quality assessment / Risk of bias analysis: Two researchers independently screened all the retrieved articles, first one by one, based on title and abstract, and then later on for full-text detailed reading. Finally, additional screening of relevant studies is performed based on the references of the identified included studies. The two lists of literature obtained above will be discussed in our team to integrate and resolve differences.

Strategy of data synthesis: Because the included outcome measurements were continuous data, as well as unit differences, we used standard mean difference (SMD) and $95 \%$ confidence interval $(\mathrm{Cl})$ for the analysis. Heterogeneity of the included studies was assessed by 12 , which was considered as low, moderate and high heterogeneity when the value of $\mathbf{I 2}$ was $25 \%, 50 \%$ and $75 \%$, respectively.

Subgroup analysis: Outcome measurements were analyzed in subgroups according to stem cell source or bone trabeculae assessment methods.

Sensitivity analysis: Sensitivity analysis is also usually performed in meta-analysis to 
assess the stability of the results of the pooled literature analysis.

Country(ies) involved: China.

Keywords: Stem cell; Osteoporosis; ovariectomized; bone mineral density; meta-analysis.

Contributions of each author: Author 1 - Zhencheng Xiong. Author 2 - Ping Yi.

Author 3 - Chi Zhang. 